\title{
Betulin exhibits anti-inflammatory activity in LPS-stimulated macrophages and endotoxin-shocked mice through an AMPK/AKT/Nrf2-dependent mechanism
}

\author{
Xinxin $\mathrm{Ci}^{1}$, Junfeng Zhou ${ }^{2}$, Hongming Lv ${ }^{1}$, Qinlei $\mathrm{Yu}^{1}$, Liping Peng ${ }^{*, 1}$ and Shucheng Hua ${ }^{*, 1}$
}

Continued oxidative stress can lead to chronic inflammation, which in turn could mediate most chronic diseases including cancer. Nuclear factor erythroid 2-related factor (Nrf2), a critical transcriptional activator for antioxidative responses, has envolved to be an attractive drug target for the treatment or prevention of human diseases. In the present study, we investigated the effects and mechanisms of betulin on Nrf2 activation and its involvement in the lipopolysaccharide (LPS)-triggered inflammatory system. In macrophages, betulin activated the Nrf2 signaling pathway and increased Nrf2-targeted antioxidant and detoxifying enzymes, including NADPH, quinine oxidoreductase 1 (NQO1), heme oxygenase-1 (HO-1), $\gamma$-glutamyl cysteine synthetase catalytic subunit (GCLC) and modifier subunit (GCLM) in a dose and time dependent manner. Importantly, we found betulin-induced activation of Nrf2 is AMPK/AKT/GSK3 $\beta$ dependent, as pharmacologically inactivating AMPK blocked the activating effect of betulin on AKT, GSK3 $\beta$ and Nrf2. Furthermore, betulin attenuated LPS-induced inflammatory mediators (iNOS and COX-2) and MAPK inflammatory signaling pathway. The effect of betulin on HO-1 and NQO1 upregulation, iNOS and COX-2 the downregulation, and survival time extension was largely weakened when Nrf2 was depleted in vitro and in vivo. Our results demonstrate that the AMPK/AKT/Nrf2 pathways are essential for the anti-inflammatory effects of betulin in LPS-stimulated macrophages and endotoxin-shocked mice. Cell Death and Disease (2017) 8, e2798; doi:10.1038/cddis.2017.39; published online 18 May 2017

Oxidative stress is a condition in which generation of reactive oxygen species (ROS) exceeds the capacity of the antioxidant defense system. Extensive research during the last two decades has revealed the mechanism by which continued oxidative stress can lead to inflammation, which in turn could mediate most chronic diseases including cancer. ${ }^{1}$ As a major component of bacterial cell walls, LPS activates the toll-like receptor-4 (TLR4) complex on host cells to dramatically increase the levels of reactive oxygen species (ROS) and initiates the systemic inflammatory response that accompanies sepsis. ${ }^{2}$ Correspondingly, inhibiting the production of intracellular ROS can suppress intracellular proinflammatory signals. ${ }^{3}$ Therefore, modulators of redox balance, considered the key regulators of inflammatory responses, and the antioxidant defense system have become major targets for inflammation research.

Nuclear factor erythroid 2-related factor 2 (Nrf2) is a transcription factor that regulates an adaptive cellular defense response to various stresses, including oxidative, proteotoxic, and metabolic stresses, as well as inflammation. ${ }^{4}$ It plays an imperative role in cellular redox homeostasis and coordinated induction of over 250 genes, including those encoding antioxidant and phase 2 detoxifying enzymes and related proteins, such as NADPH, quinine oxidoreductase 1 (NQO1), heme oxygenase-1 (HO-1), r-glutamyl cysteine synthetase catalytic subunit (GCLC) and modifier subunit (GCLM). ${ }^{5}$
Activating this pathway is one of the main defense mechanisms against oxidative stress. ${ }^{6} \mathrm{Nrf2}$ is held in the cytoplasm as an inactive complex bound to Keap1, a repressor molecule that facilitates Nrf2 ubiquitination. ${ }^{7}$ Although the mechanism by which Nrf2 is liberated from the Keap-Nrf2 complex remains to be established, recent studies have suggested that the phosphorylation of Nrf2 at serine and threonine residues by upstream kinases, such as protein kinase $\mathrm{C}$, phosphatidylinositol-3-kinase/Akt, and mitogen-activated protein kinase, facilitate the release of Nrf2 from Keap1 and its subsequent translocation, followed by the initiation of antioxidative cascades. ${ }^{8}$ AMP-activated protein kinase (AMPK), a heterotrimeric serine/threonine kinase, is an important energy sensor of cellular metabolism in response to metabolic stress, such as oxidative stress, inflammation, and neurodegeneration. ${ }^{9,10}$ Accordingly, AMPK activation exerts anti-inflammatory effects, regulates catabolism and anabolism and improves redox balance. ${ }^{11-14}$ Lee and $\mathrm{Kim}^{15}$ recently demonstrated that AMPK can stimulate the Nrf2 signaling pathway in Raw264.7 cells, but the underlying mechanisms of the AMPK-mediated antioxidant response remain unclear. Previous studies have suggested that AMPK activated the PI3K/Akt signaling pathway. ${ }^{16}$ Moreover, AMPK also increases inhibitory phosphorylation of glycogen synthase kinase 3 beta (GSK3 $\beta),{ }^{17}$ which contributes to mitochondrial protection against iron-induced oxidant stress. ${ }^{18}$ In addition,

${ }^{1}$ Institute of Translational Medicine, Department of Respiratory Medicine, The First Hospital, Jilin University, Changchun 130001, China and ${ }^{2}$ Department of Dermatology and Venereology, The First Hospital of Jilin University, Changchun, Jilin Province, China

${ }^{*}$ Corresponding author: Shucheng hua or Liping Peng, Department of Respiratory Medicine, The First Hospital, Jilin University, Changchun 130001, China. Tel: 043188783044 or 0431-88782102; Fax: +86 431 88783044; E-mail: m15904300579@163.com or plp640317@163.com

Received 07.12.16; revised 11.1.17; accepted 12.1.17; Edited by H-U Simon 
several lines of evidence have identified GSK3 $\beta$ as a novel regulator of Nrf2, which suggests that Nrf2 may cooperate with the AMPK/Akt/GSK3 $\beta$ signaling networks. ${ }^{19,20}$

Betulin (lup-20(29)-ene-3 $\beta$, 28-diol), a triterpene extracted from birch tree bark, has been reported to have diverse pharmacological activities, such as anti-inflammatory, antibacterial and antiviral activities. ${ }^{21}$ Although betulin may exhibit protective effects on cognitive decline in STZ-induced diabetic rats through the $\mathrm{HO}-1 / \mathrm{Nrf}-2$ pathway and inhibit alcoholic liver injury via activation of AMPK, no information about the potential for betulin involvement in the interactions and temporal relationship between AMPK and Nrf2 pathway in the inflammatory system exists. ${ }^{22,23}$ In the present study, utilizing LPS-stimulated macrophages and endotoxinshocked mice, we aimed to investigate the effects of betulin on inflammatory stress and the functional interaction between Nrf2 and AMPK pathways. Our results suggested that the anti-inflammatory role of betulin depends on Nrf2 activation and is primarily involved with the AMPK/AKT/GSK3 $\beta$ pathways in LPS-stimulated macrophages and endotoxinshocked mice.

\section{Results}

Effects of betulin on Nrf2 translocation, Keap1 and antioxidant enzyme expression. The Keap1-Nrf2 signaling axis is a master regulator that regulates the ARE-driven expression of phase II detoxifying and antioxidant enzymes, such as HO-1, NQO1 and $\gamma$-glutamate cysteine ligase subunit (GCLS). As shown in Figures 1a and b, treatment with betulin increased Nrf2 translocation from cytoplasm to nucleus and downregulated the expression of the Keap1 protein in a dose-dependent manner. Moreover, we further assessed the a

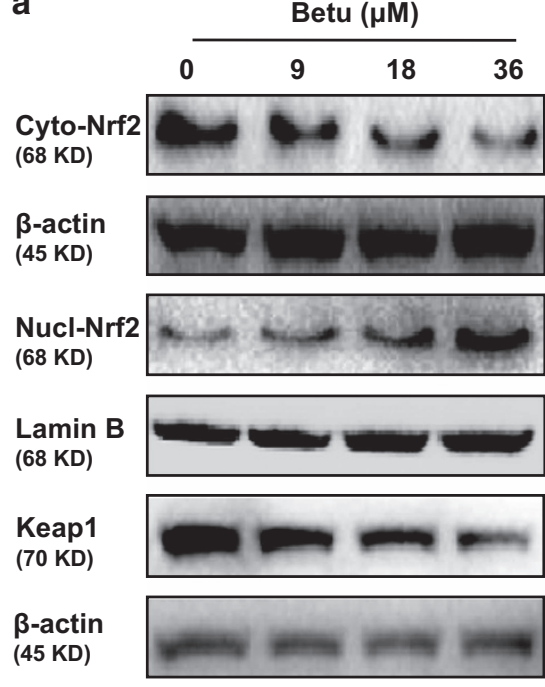

b

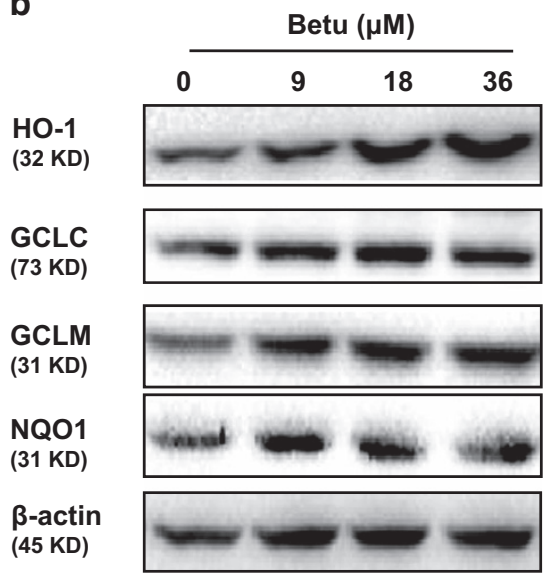

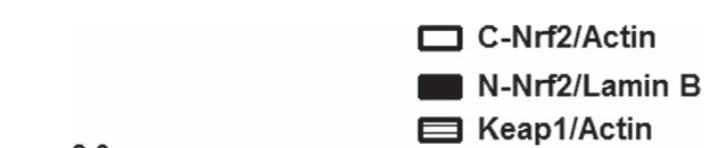

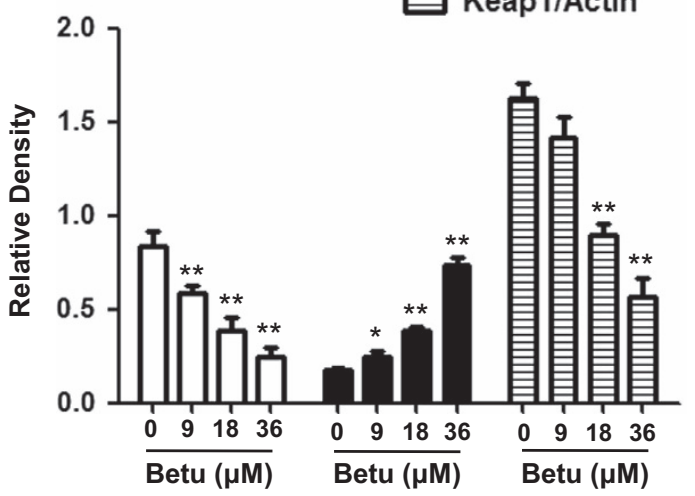

$\square$ HO-1 /Actin

GCLC/Actin

曰GCLM/Actin

IIII NQ01/Actin

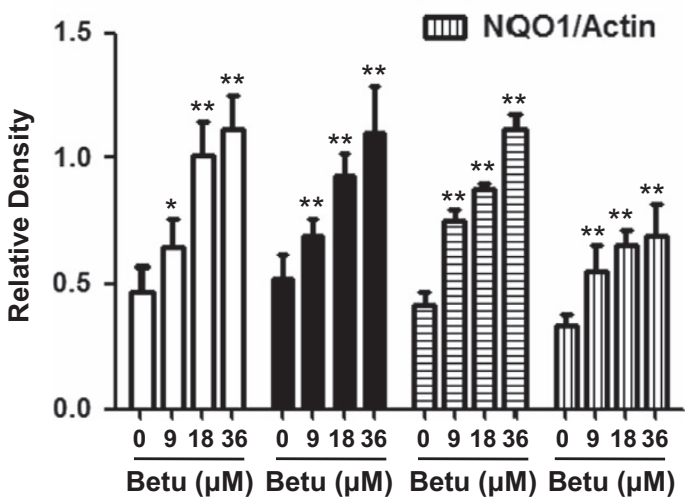

Figure 1 Effects of betulin on Nrf2 translocation, Keap1 and antioxidant enzyme expression. (a) RAW 264.7 cells were plated in six-well culture plates and treated with betulin $(9,18$ and $36 \mu \mathrm{M})$ for $6 \mathrm{~h}$. Nuclear and cytoplasmic extracts from RAW 264.7 cells were prepared for detecting Nrf2 and total protein extracts for detecting Keap1. (b) RAW 264.7 cells were treated with betulin $(9,18$ and $36 \mu \mathrm{M})$ for $18 \mathrm{~h}$ and total protein extracts were prepared for detecting antioxidant enzymes. Quantifications were performed by densitometric analysis, and Lamin B and $\beta$-actin acted as an internal control. A representative western blot is shown in the left panel. Shown in the right panel are means \pm S.E.M. of three independent experiments. ${ }^{*} P \leq 0.05,{ }^{* \star} P \leq 0.01$ versus the control group 
expression of antioxidant enzymes and demonstrated that HO-1, NQO1,GCLC and GCLM were upregulated by betulin in a dose-dependent manner (Figures $1 \mathrm{c}$ and $\mathrm{d}$ ).

Effects of betulin on the AMPK/AKT/GSK3 $\beta$ and MAPK pathway in RAW 264.7 cells. Recent reports have suggested that phosphatidylinositol 3-kinase (PI3K), c-Jun $\mathrm{N}$-terminal kinase (JNK) and extracellular signal-regulated protein kinase (ERK) are assumed to facilitate the release of Nrf2 from Keap1 and its subsequent translocation. We examined the activation of betulin on the AKT and MAPK pathway in RAW264.7 cells. The results indicated that betulin slightly decreased JNK and ERK, slightly increased p38, but significantly increased AKT phosphorylation in a dosedependent manner (Figure 2). Moreover, previous studies suggested that AMPK activated the PI3K/Akt signaling pathway and increased inhibitory phosphorylation of glycogen synthase kinase 3 beta (GSK3 $\beta$ ). We examined the effect of betulin on AMPK and GSK3 $\beta$ phosphorylation and demonstrated that betulin activated AMPK and GSK3 $\beta$ phosphorylation in a dose-dependent manner.

Involvement of the AMPK/AKT/GSK3 $\beta$ pathway in Nrf2 nuclear translocation by Betulin. To further determine the upstream signaling pathway involved in betulin-mediated Nrf2 activation, we investigated the effects of compound $\mathrm{C}$, a specific inhibitor of AMPK, on Nrf2 nuclear translocation. As shown in Figure 3, compound $\mathrm{C}$ dramatically inhibited AMPK, AKT and GSK3 $\beta$ phosphorylation and Nrf2 nuclear translocation, which suggested that betulin modulated Nrf2 nuclear translocation via the activation of the AMPK/AKT/GSK3 $\beta$ signaling pathway in RAW264.7 cells.

Betulin activated anti-inflammatory and antioxidant reactions in LPS-stimulated macrophages. Elevation of inflammatory gene expression is a well-known response of macrophages to LPS stimulation; therefore, we assessed the suppressive effect of betulin on the representative

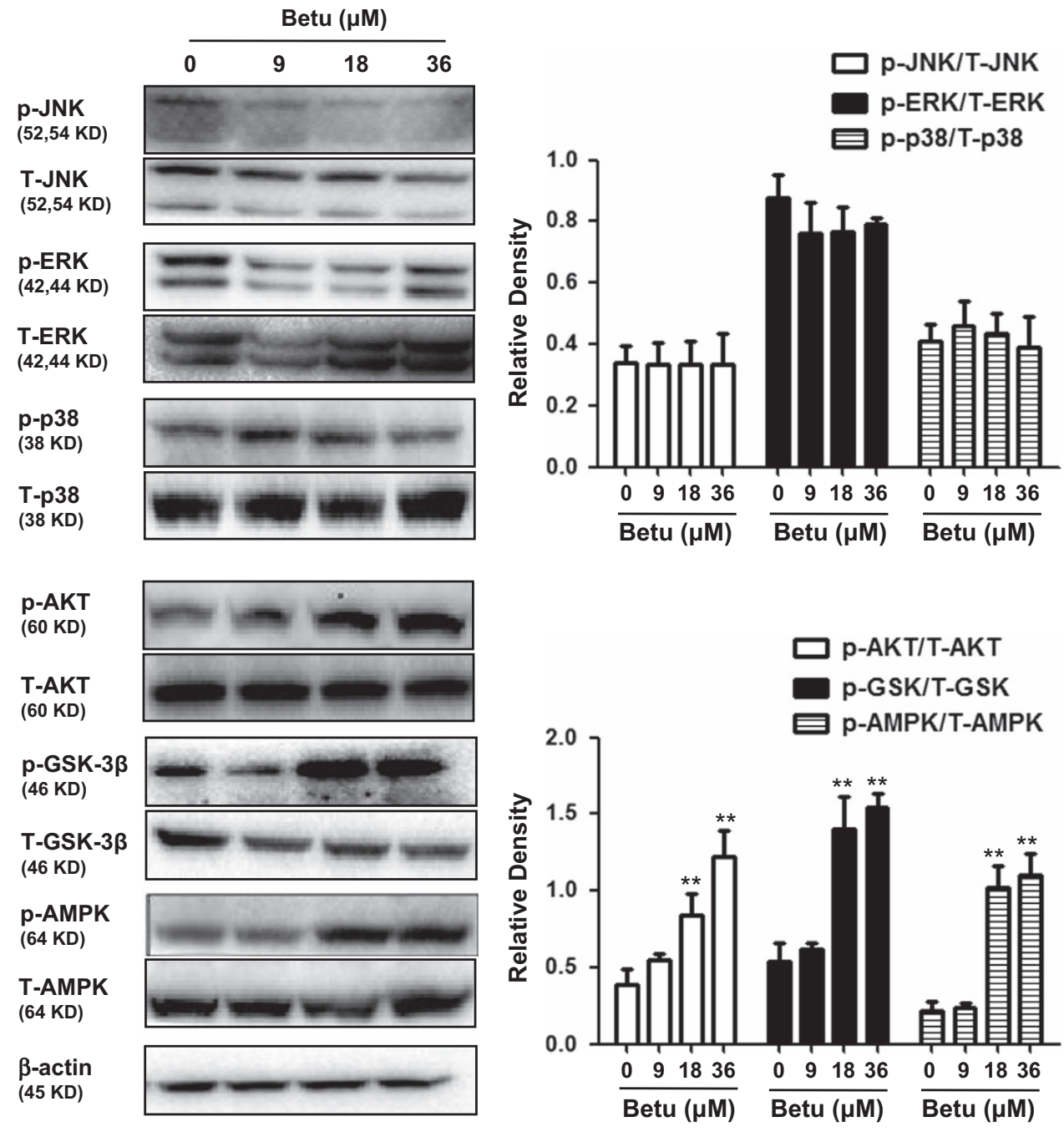

Figure 2 Effects of betulin on AMPK/AKT/GSK3 $\beta$ and MAPK pathways in RAW 264.7 cells. RAW 264.7 cells were treated with betulin $(9,18$ and $36 \mu$ M) for $6 \mathrm{~h}$ and then immunoblotted with specific antibodies. Quantifications were performed by densitometric analysis, and $\beta$-actin acted as an internal control. A representative western blot is shown in the left panel. Shown in the right panel are means \pm S.E.M. of three independent experiments. ${ }^{\star *} P \leq 0.01$ versus the control group 


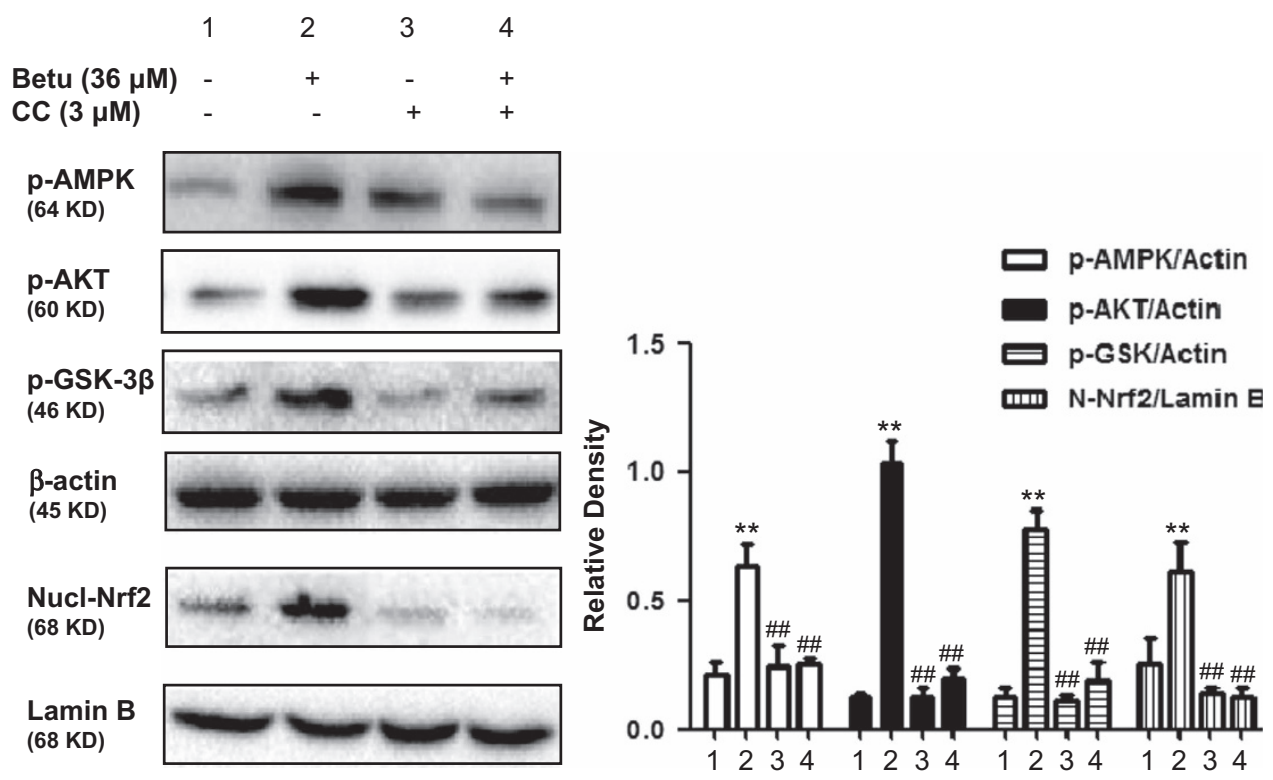

Figure 3 Involvement of the AMPK/AKT/GSK3 $\beta$ pathway in Nrf2 nuclear translocation by Betulin. Cells were treated with $3 \mu \mathrm{M}$ compound $\mathrm{C}$ (Comp.C) for $18 \mathrm{~h}$ before treatment with betulin $(36 \mu \mathrm{M})$ for $6 \mathrm{~h}$. Cell lysates were immunoblotted for the phosphorylation of AMPK, Akt, GSK3 $\beta$ and Nrf2. A representative western blot is shown in the left panel. Shown in the right panel are means \pm S.E.M. of three independent experiments. ${ }^{\star *} P \leq 0.01$ versus the control group; ${ }^{\# \#} P \leq 0.01$ versus the betulin group

inflammatory genes. As shown in Figure 4a, betulin significantly suppressed LPS-induced expression of iNOS and COX-2. Furthermore, Nrf2-regulated antioxidant enzymes play a vital role in the anti-inflammatory response. As shown in Figure 4a, LPS had almost no effect on the expression of $\mathrm{HO}-1$ and NQO1, but betulin significantly upregulated expression of these genes. Furthermore, a burst of production of free radicals is known to accompany the inflammatory response; therefore we detected the effect of betulin on ROS production. As shown in Figures $4 \mathrm{~b}$ and $\mathrm{c}$, betulin alone did not affect ROS production, but significantly inhibited LPS-induced ROS production. Moreover, protecting mitochondria from free radicals is an important aspect of the antioxidative and anti-inflammatory responses. To address the effect of betulin on mitochondrial protection, we first assayed mitochondrial membrane potential using a fluorescent mitochondrial dye, JC-1. As shown in Figure 4d, MMP was greatly degraded by LPS, showing obvious green fluorescence, while betulin effectively restored the MMP.

Knockout of Nrf2 abolished the protective effects of betulin on inflammatory and oxidant reactions in LPSstimulated macrophages. Antioxidation is important for the anti-inflammatory response, with Nrf2-regulated antioxidant enzymes being vital to this antioxidative response. Therefore, we used RAW264.7 $\mathrm{Nrf}^{-/-}$cells to investigate whether the anti-inflammatory and antioxidant effects of betulin were mediated by Nrf2. Compared with control cells, $\mathrm{Nrf2}^{-/-}$cells markedly suppressed the Nrf2, $\mathrm{HO}-1$ and NQO1 protein expression induced by betulin (Figures 5a, c and d). Furthermore, our results showed that the protective effects of betulin on iNOS, COX-2 and ROS production were attenuated in $\mathrm{Nrf}^{-1-}$ cells (Figures $5 \mathrm{~b}$, e and f).
Betulin regulated anti-inflammatory and antioxidant signaling pathways in LPS-stimulated macrophages. Nrf2 translocation from the cytoplasm to the nucleus is a dispensable step for Nrf2 activation. The nuclear distribution of Nrf2 protein was determined by western-blot assay, with the results showing that LPS has no effect on Nrf2 protein in the nuclear fraction, but that betulin alone or together with LPS increased nuclear Nrf2 protein expression (Figure 6). $\mathrm{HO}-1$, an antioxidant enzyme regulated by Nrf2, was shown to be upregulated by betulin alone or together with LPS. Some typical signaling pathways, such as MAPK, NF- $k$ B and $\mathrm{AKT}$, are involved in the inflammatory response; therefore, we analyzed the effects of betulin on LPS-stimulated inflammatory protein expression. Our results indicated that the protein expression of iNOS, COX-2 and HMGB1 and the levels of JNK, ERK, p38 and AKT phosphorylation dramatically increased in RAW264.7 cells exposed to LPS, whereas the expression of all of these proteins decreased following betulin pretreatment.

Suppressive effects of betulin on LPS-induced endotoxin shock is Nrf2 dependent in mice. The dependency of the anti-inflammatory role of betulin on Nrf2 was further assessed in endotoxin-shock mice. First, as shown in Figure 7a, for $\mathrm{Nrf}^{+/+}$mice (WT), the median survival times in the control group and in the betulin-treated group were 36 and $96 \mathrm{~h}$, respectively, suggesting that betulin has significant protective effects. However, for Nrf2 $2^{-1-}$ mice, the median survival times both in the control group and in the betulin-treated group were $60 \mathrm{~h}$. Second, for WT mice, the final survival rate was $0 \%$ (control group) versus $40 \%$ (betulin-treated group). Meanwhile, for $\mathrm{Nrf}^{-1-}$ mice, the final survival rate was $20 \%$ (control group) versus $30 \%$ (betulin-treated group). Since 
a
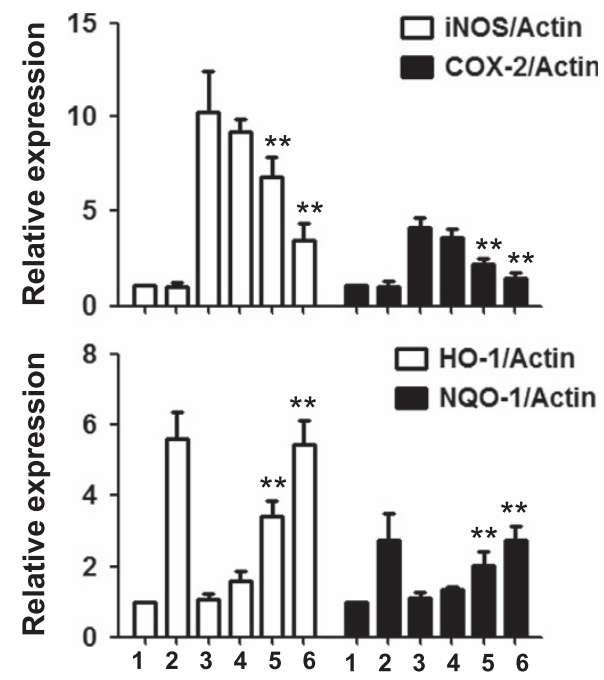

b
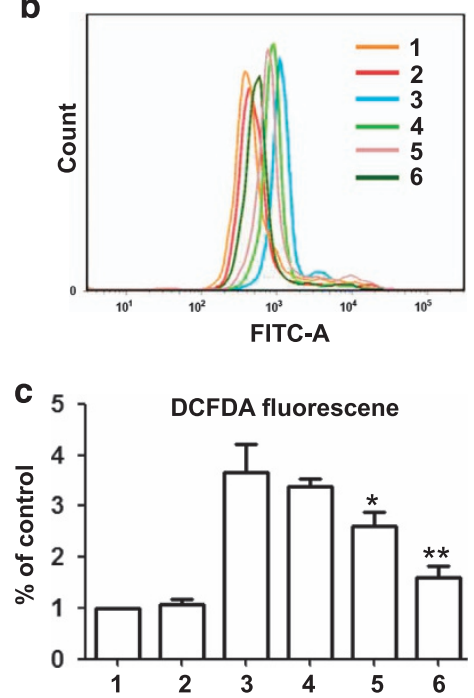

d
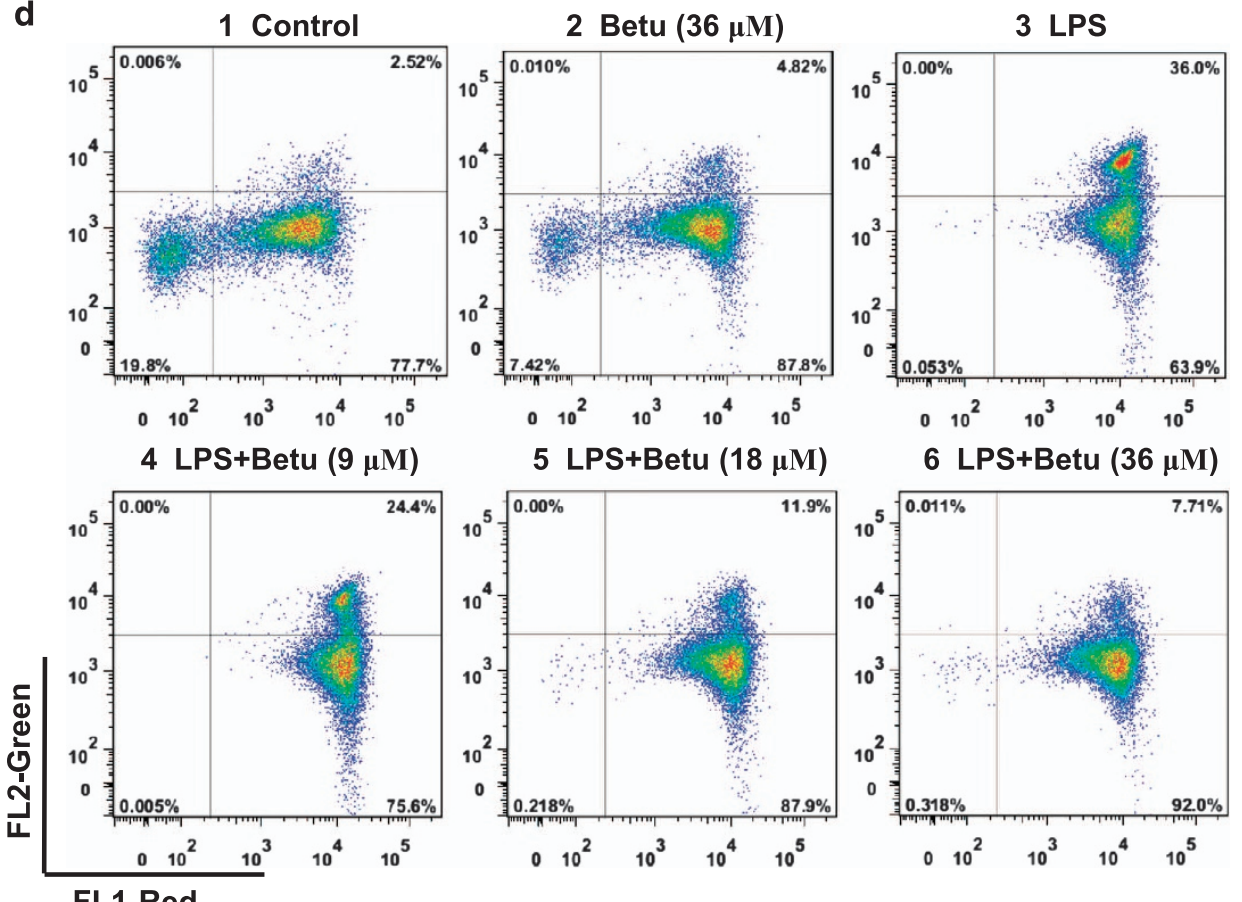

Figure 4 Effects of betulin on anti-inflammatory and antioxidant reactions in LPS-stimulated macrophages. RAW264.7 cells were plated in 12-well plates, preincubated with betulin $(9,18$ and $36 \mu \mathrm{M})$ for $1 \mathrm{~h}$, and then challenged with LPS ( $500 \mathrm{ng} / \mathrm{ml}$ ) for $18 \mathrm{~h}$. (a) The expression of proinflammatory genes (iNOS and COX-2) and antioxidant enzymes ( $\mathrm{HO}-1$ and NQO1) were detected by quantitative real-time PCR. (b and $\mathbf{c}$ ) The cells were incubated with $50 \mu \mathrm{M}$ of DCFH-DA for 40 min, and DCF fluorescence intensities were detected by flow cytometry. (d) The cells were washed with PBS and incubated with JC-1 $(10 \mu \mathrm{g} / \mathrm{ml})$ at $37^{\circ} \mathrm{C}$ in the dark for $30 \mathrm{~min}$. JC- 1 fluorescence was quantified through flow cytometry. ${ }^{*} P \leqslant 0.05,{ }^{* \star} P \leqslant 0.01$ versus the LPS group

lung injury is remarkable in LPS-shocked mice, we isolated RNA from lung tissue. As shown in Figures $7 b-d$, betulin significantly increased mRNA expression of antioxidant genes (HO-1 and NQO1), decreased the mRNA expression of anti-inflammatory genes (iNOS and COX-2) and decreased $I_{\kappa} \mathrm{B} a$ phospholation. However, both the antioxidant and anti-inflammatory effects of betulin were abrogated or attenuated in $\mathrm{Nrf}^{-/-}$mice.

\section{Discussion}

Extensive research during the last two decades has revealed that oxidative stress can activate inflammatory signaling pathways such as NF- $\mathrm{B}$ and $\mathrm{MAPK}$, which regulate cytokines, chemokines, cyclooxygenase-2 (COX-2), inducible nitric oxide synthase (iNOS), and anti-inflammatory molecules. $^{24}$ Continued oxidative stress can lead to 
a

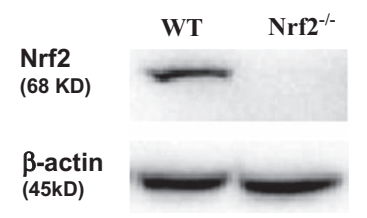

C

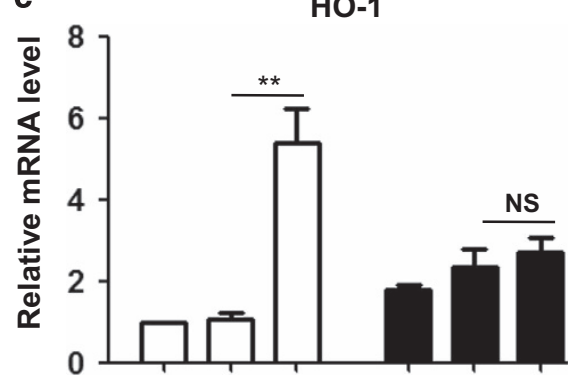

e

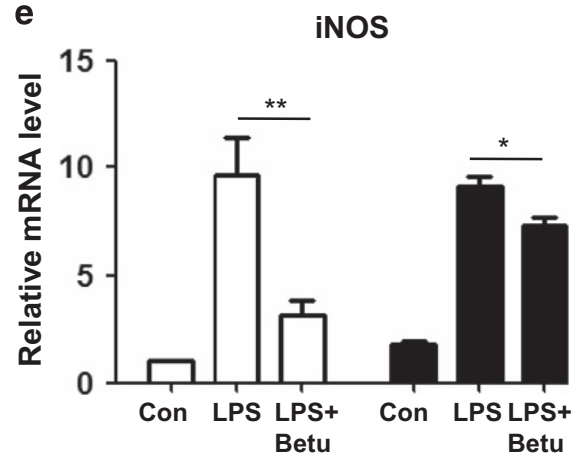

b

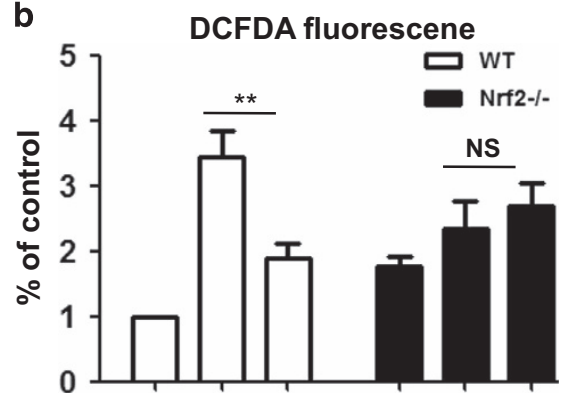

d

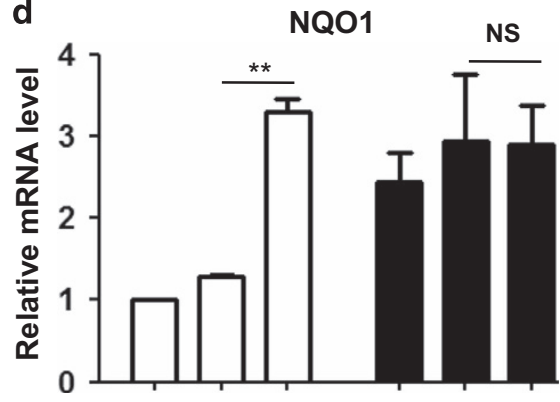

f

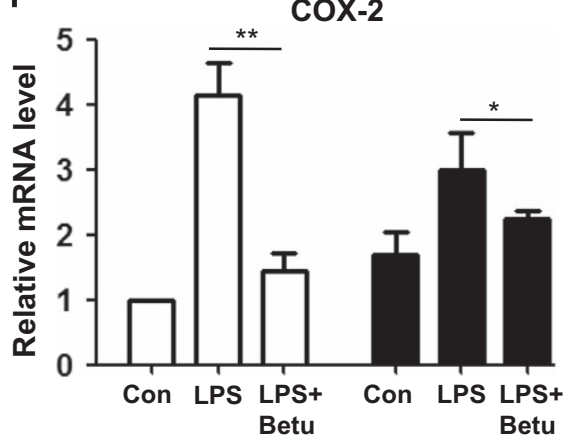

Figure 5 Knockout of Nrf2 abolished the protective effects of betulin on inflammatory and oxidant reactions in LPS-stimulated macrophages. (a) Normal (WT) and Nrf2 knockout ( $\mathrm{Nrf}^{-1-}$ ) RAW264.7 cells were cultured with betulin ( $\left.36 \mu \mathrm{M}\right)$ for $6 \mathrm{~h}$ to detect Nrf2 expression. (b) WTand Nrf2 ${ }^{-1-}$ cells were cultured with or without betulin (36 $\mu \mathrm{M}$ ) for $1 \mathrm{~h}$ and then challenged with LPS (500 ng/ml) for $18 \mathrm{~h}$; then, the cells were incubated with $50 \mu \mathrm{M}$ of DCFH-DA for $40 \mathrm{~min}$, and DCF fluorescence intensities were detected by flow cytometry. (c-f), WT cells and Nrf2 ${ }^{-1-}$ cells were cultured with or without betulin $(36 \mu \mathrm{M})$ for $1 \mathrm{~h}$ and then challenged with LPS $(500 \mathrm{ng} / \mathrm{ml})$ for $18 \mathrm{~h}$; then, the expression of proinflammatory genes (iNOS and COX-2) and antioxidant enzymes (HO-1 and NQO1) were detected by quantitative real-time PCR. All of the data shown represent the average of three independent experiments. ${ }^{*} P \leq 0.05,{ }^{* *} P \leq 0.01$ versus the LPS group; NS, no significant difference

inflammation, which can further mediate most chronic diseases including cancer, diabetes, cardiovascular and pulmonary diseases. ${ }^{25}$ The nuclear factor erythroid 2-related factor 2 (Nrf2) pathway plays an imperative role in cellular redox homeostasis, and the activation of this pathway is one of the main defense mechanisms against oxidative stress. In the present study, we found that betulin has anti-inflammatory potential via its activation of the Nrf2 pathway, and this activation of Nrf2 is AMPK-dependent in a LPS-challenged inflammatory response.

Nrf2 can prevent cellular damage associated with various types of injury in many different cell types. Nrf2-mediated protection depends on the expression of detoxification and antioxidant genes such as HO-1, NQO1, GCLC and GCLM. ${ }^{5}$ Previous studies also link the activation of PI3K, PKC and MAPK signaling pathways to the regulation of Nrf2-related antioxidant gene expression. ${ }^{26}$ In the present study, we demonstrated that betulin regulation of Nrf2 and antioxidant gene expression is mediated through degradation of Keap1 and activation of Akt and AMPK; pretreatment with AMPK spefific inhibitors (Compound $\mathrm{C}$ ) attenuated the activation of AKT, Akt-mediated inhibitory phosphorylation of GSK3 $\beta$ and consequently Nrf2 which induced by betulin. Taken together, these results suggested that betulin has antioxidant potential via its activation of the AMPK/AKT/GSK3 $\beta / N r f 2$ and Nrf2/ Keap1 pathways in RAW264.7 cells.

Many studies have reported on the therapeutic benefits of betulin in experimental animal models. The most widely studied effect of betulin is its anti-inflammatory activity in various inflammatory model including COPD, alcoholic liver injury, mammary gland inflammatory injury and chronic dermal inflammation. ${ }^{23,27,28}$ It has been reported that betulin decreased proinflammatory cytokines via inhibiting NF- $\kappa \mathrm{B}$ and MAPK signaling pathways. ${ }^{29}$ Given this situation and the 


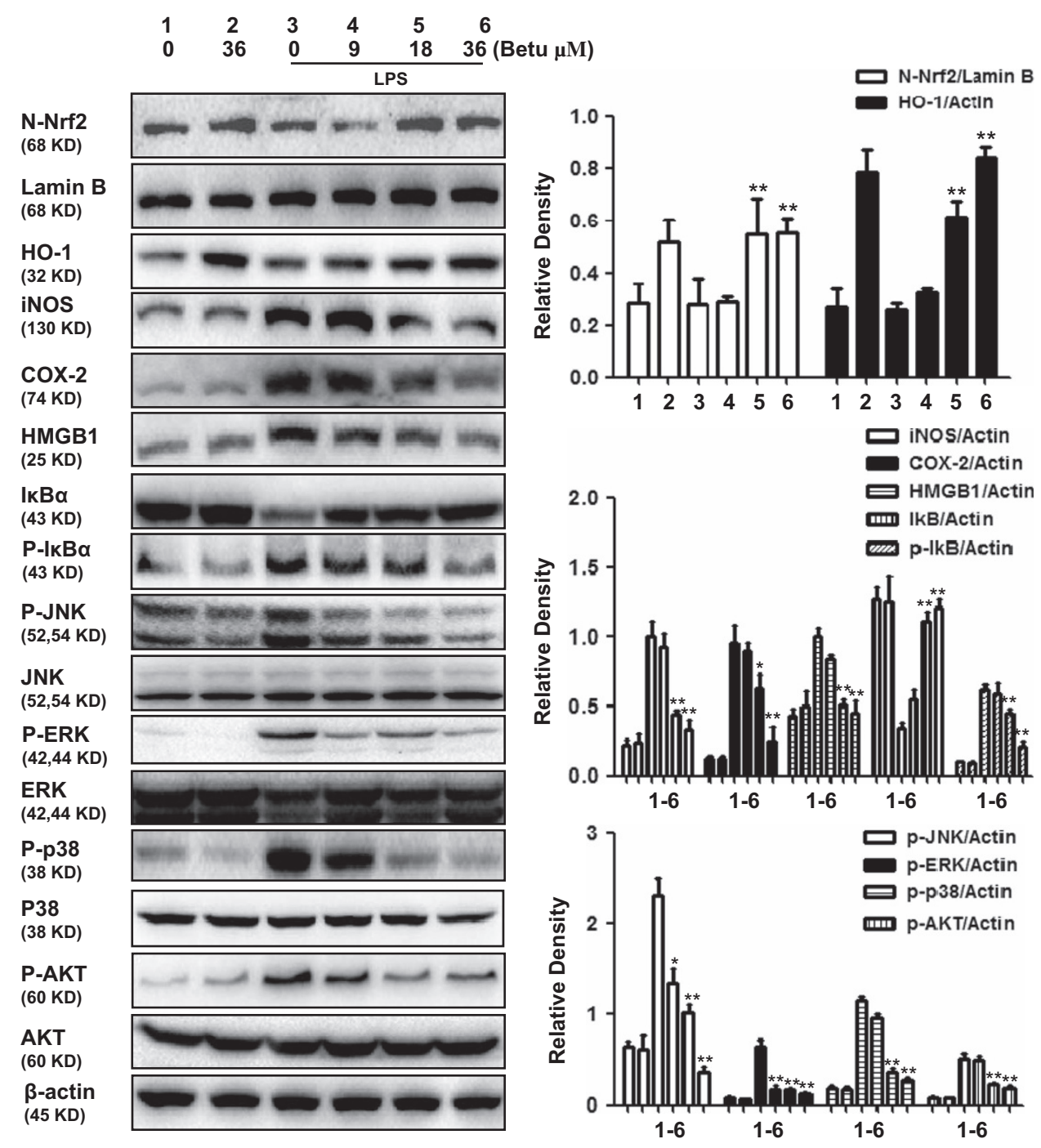

Figure 6 Effect of betulin on anti-inflammatory and antioxidant signaling pathways in LPS-stimulated macrophages. RAW264.7 cells were plated in 6-well plates, preincubated with betulin $(9,18$ and $36 \mu \mathrm{M})$ for $1 \mathrm{~h}$, and then challenged with LPS (500 ng/ml) for $18 \mathrm{~h}$. Cell lysates were immunoblotted with different antibodies. A representative Western blot is shown in the left panel. Shown in the right panel are means \pm S.E.M. of three independent experiments. ${ }^{*} P \leq 0.05,{ }^{* *} P \leq 0.01$ versus the LPS group

promising importance of the relationship between oxidative stress and inflammation, we posed two specific questions: is there a functional link between the anti-inflammatory and antioxidant effects of betulin? If there is, how do they work together to inhibit inflammation? On the basis of these questions, we assessed the effect of betulin on representative inflammatory genes (iNOS and COX-2) and antioxidant enzymes (HO-1 and NQO1) in LPS-stimulated RAW264.7 cells. Our results demonstrated that betulin significantly suppressed the LPS-induced expression of iNOS and COX-2 but upregulated $\mathrm{HO}-1$ and NQO1 gene expression. Furthermore, LPS-induced ROS production and MMP degradation were significantly inhibited by betulin. These investigations indicated that betulin possessed anti-inflammatory and antioxidant effects, whereas these effects were attenuated or abolished in RAW264.7 Nrf2 ${ }^{-/}$cells, suggesting that the compensatory upregulation of Nrf2-mediated expression of antioxidant genes is induced by betulin to protect against LPS-induced inflammation.

On the basis of above outcome, we further explored the mechanisms involved in the anti-inflammatory effects of betulin. Several reports have shown that the LPS signaling cascade leading to iNOS and COX2 expression in macrophages is dependent on the phospholation of the members of the MAPKs family: p38, ERK1/2 and NF- $\kappa$ B p65 translocation from the cytoplasm to the nucleus. ${ }^{30,31}$ Under resting conditions, NF- $k \mathrm{~B}$ is held inactive by $I_{\kappa} \mathrm{B}$. The phosphorylation of $I_{\kappa} \mathrm{B}$ results in $\mathrm{I}_{\kappa} \mathrm{B}$ degradation and further NF- $\kappa \mathrm{B}$ dissociation, which leads to the induction of pro-inflammatory cytokines and mediators. ${ }^{32}$ Protein expression was accordingly measured in western blot assays. Our results indicate that LPS markedly induced the inflammatory protein expression but did not affect $\mathrm{Nrf2}$ and $\mathrm{HO}-1$ expression, which was regulated by betulin pretreatment. We further investigated the 
a

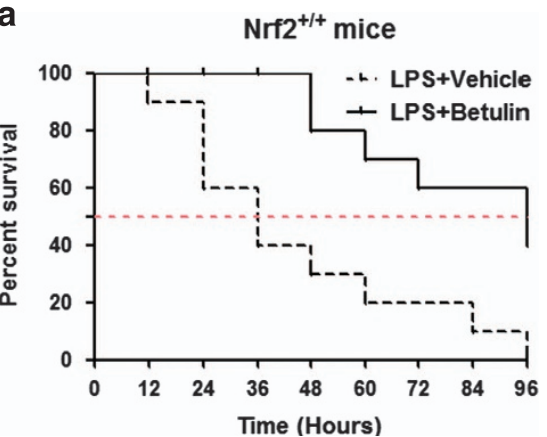

b

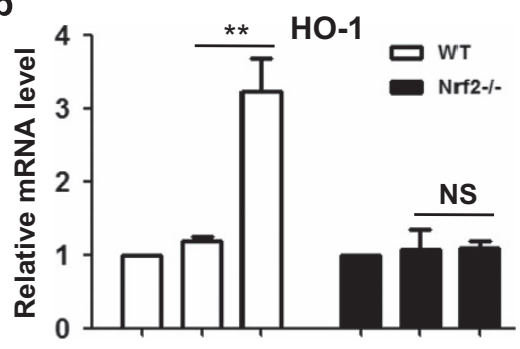

C

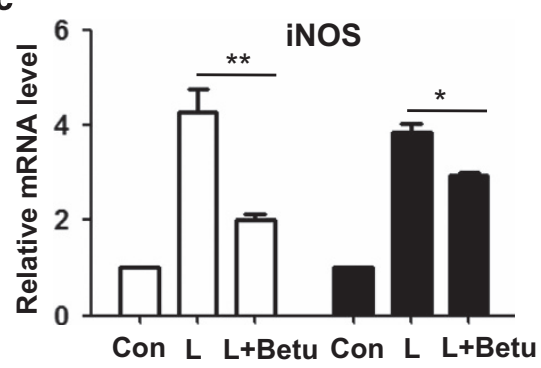

d

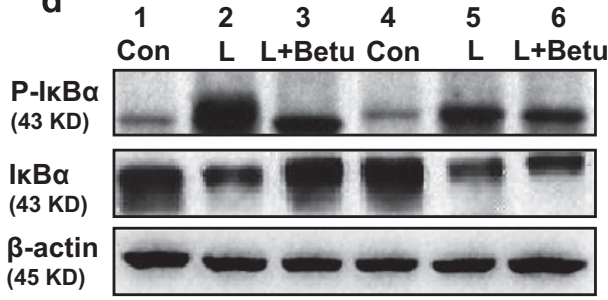

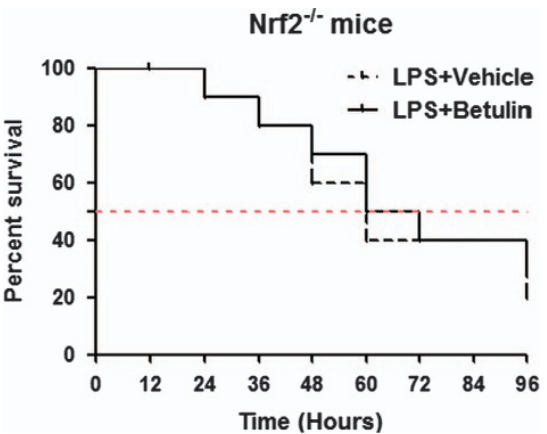
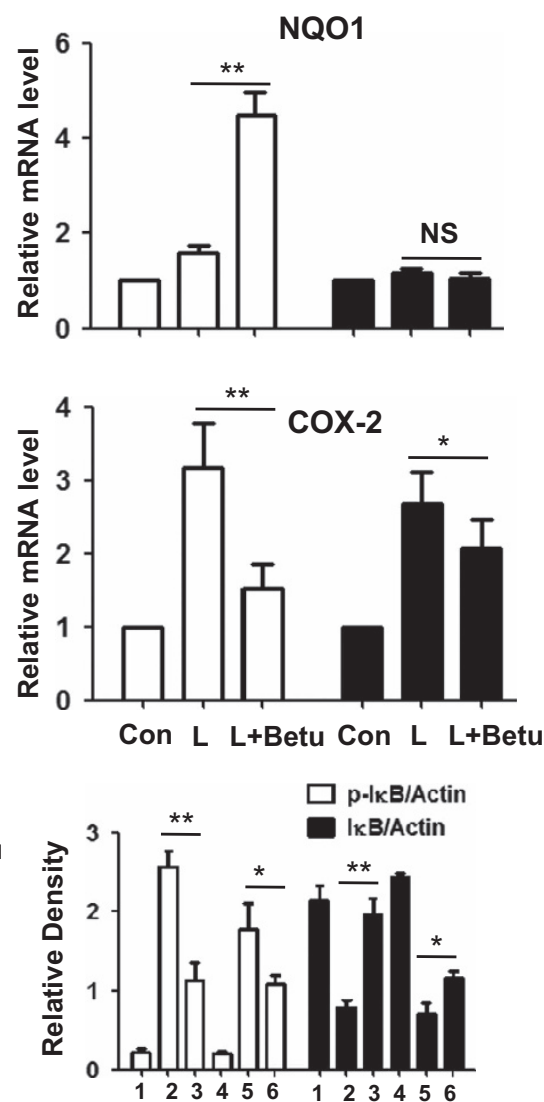

Figure 7 Suppressive effects of betulin on LPS-induced endotoxin shock are Nrf2 dependent in mice. (a) Effect of betulin on the survival rate of wt or Nrf2 ${ }^{-1}{ }^{-}$mice. Mice were treated with betulin (12 mg/kg) and LPS (40 mg/kg) as described in the Material and Methods $(n=10)$; the survival rates are presented as survival curves. (b-d), Effect of betulin on the genes and protein expression in the lung tissue of mice. $P^{\star} \leq 0.05, P^{* *} \leq 0.01$ versus the LPS group; NS, no significant difference

Nrf2 pathway involved in the anti-inflammatory effect of betulin against LPS in vivo. For $\mathrm{Nrf}^{+/+}$endotoxin-shocked mice, betulin prevented the mortality associated with LPS challenge, but the anti-inflammatory role of betulin was clearly attenuated in Nrf2-deficient mice. Surprisingly, $\mathrm{Nrf}^{-/-}$mice were less susceptible to LPS and developed less severe endotoxinshocked phenotypes than $\mathrm{Nrf}^{+/+}$mice did, as determined by a slight decrease in inflammation and an increased survival rate. Moreover, consistent with in vitro results, betulin abolished the upregulation of $\mathrm{HO}-1$ and NQO1 but only attenuated the downregulation of iNOS and COX-2 expression in the lung tissue of $\mathrm{Nrf}^{-1-}$ mice. In addition, the inhibitory effect of betulin on NF- $\mathrm{BB}$, as a regulator of iNOS and COX-2 expression, has been attenuated in the lung tissue of $\mathrm{Nrf}^{-/-}$ mice. Taken together, our results support that the inhibitory effect of betulin in inflammation is, at least partially, based on a mechanism underlying the suppression of proinflammatory cascades on one hand, and on the activation of Nrf2-mediated antioxidative cascades on the other hand.

In conclusion, our present study for the first time demonstrates that betulin exhibits anti-inflammatory activity in LPSstimulated macrophages and endotoxin-shocked mice through activation of Nrf2 and that AMPK/AKT/GSK3 $\beta$ works upstream of Nrf2. This finding provides an innovative platform 
to explore the mechanism underlying anti-inflammatory and antioxidant effects and further contributes to the development of new therapeutic approaches for inflammatory diseases.

\section{Materials and Methods}

Reagents. Betulin (analytical grade, purity $\geq 98 \%$ ) was obtained from the National Institute for the Control of Pharmaceutical and Biological Products (Beijing, China). LPS and LY294002 were purchased from the Sigma Chemical Co. (St. Louis, MO, USA). Compound C (AMPK inhibitor) was obtained from MedChem Express (Monmouth, NJ, USA). Antibodies against Nrf2, HO-1, NQ01, GCLC, GCLM, iNOS, COX-2, HMGB1, KEAP1, AKT, p-AKT, GSK3 $\beta$, p-GSK3 $\beta$ Ser, ${ }^{9}$ ERK, pERK, JNK, p-JNK, p38, p-p38, AMPK, p-AMPK $\alpha$ (Thr172), I $\mathrm{I}_{\kappa} \alpha, \mathrm{P}-\mathrm{I}_{\kappa} \mathrm{B} \alpha$, Lamin B, as well as $\beta$-actin were purchased from Cell Signaling (Boston, MA, USA) or Abcam (Cambridge, MA, USA). The horseradish peroxidase-conjugated anti-rabbit and anti-mouse IgG were purchased from proteintech (Boston, MA, USA). Prime-Script RT-PCR kit and Faststart Universal SYBR Green Master were offered by Takara (Dalian, China) and Roche (Basel, Switzerland), respectively. 3'-tetraethylbenzimidazolyl-carbocyanine iodide (JC-1) were offered from Beyotime Institute of Biotechnology (Jiangsu, China).

Cells. The RAW264.7 murine macrophage cell line was obtained from the China Cell Line Bank (Beijing, China). Cells were cultured in Dulbecco's modified Eagle medium supplemented with $3 \mathrm{mM}$ glutamine, antibiotics $(100 \mathrm{U} / \mathrm{ml}$ penicillin and $100 \mathrm{U} / \mathrm{ml}$ streptomycin), and $10 \%$ heat-inactivated fetal bovine serum. The cells were maintained at $37{ }^{\circ} \mathrm{C}$ in a humidified incubator containing $5 \% \mathrm{CO}_{2}$. In all experiments, cells were allowed to acclimate for $24 \mathrm{~h}$ before any treatments.

Animals. Normal B6 mice $\left(\mathrm{Nrf}^{+/+}\right)$and $\mathrm{Nrf2}$ deficient mice $\left(\mathrm{Nrf2}^{-/-}\right)$with B6 background were provided by Beijing weitonglihua and Jackson lab. All animals were bred under specific pathogen-free conditions. All experiments were conducted according to the experimental practices and standards approved by the Animal Welfare and Research Ethics Committee at Jilin University.

CRISPR/Cas9 knockout. RAW264.7 cells were grown in 24-well plates for $16 \mathrm{~h}$, and then transfected with one plasmid expressing Cas9 with Nrf2 sgRNA and one plasmid carrying a puromycin resistance gene using Viafect transfection reagent (Promega, Madison, USA). After $36 \mathrm{~h}, 2 \mathrm{mg} / \mathrm{ml}$ puromycin was added to selected cells. Two days later, live cells were seeded in 96 -well plates at a density of one cell per well. The level of gene editing efficiency after the clonal expansion was determined by immunoblotting. To verify the edited genes, DNA sequencing was employed.

Quantitative real-time polymerase chain reaction analysis. Cells were treated with or without betulin $(9,18$ and $36 \mu \mathrm{M})$ for $1 \mathrm{~h}$, then treated with LPS $(500 \mathrm{ng} / \mathrm{ml})$ for $18 \mathrm{~h}$. Total RNA from cells was isolated by using Trizol reagent, and the manufacturer's instructions. After the concentration of RNA was determined by spectrophotometer, $1 \mu \mathrm{g}$ of RNA was converted to cDNA by Prime-Script RT-PCR kit Real-time PCR analysis was performed using the Applied Biosystems 7300 realtime PCR system and software (Applied Biosystems, Carlsbad, CA, USA). Realtime PCR was conducted in $0.2 \mathrm{ml}$ PCR tubes with forward and reverse primers and the SYBR green working solution, using a custom PCR master mix with the following conditions: $95^{\circ} \mathrm{C}$ for $10 \mathrm{~min}$, followed by 40 cycles of $95^{\circ} \mathrm{C}$ for $10 \mathrm{~s}, 60^{\circ} \mathrm{C}$ for $30 \mathrm{~s}$. The relative gene expression were analyzed by normalizing with GAPDH mRNA expression.

Western blot analysis. Cells were treated with or without betulin $(9,18$ and $36 \mu \mathrm{M})$ for $1 \mathrm{~h}$, then treated with LPS $(500 \mathrm{ng} / \mathrm{ml})$ for $18 \mathrm{~h}$. Cell homogenates were centrifuged at $3000 \times g$ for $5 \mathrm{~min}$. Nuclear and cytoplasmic fractions of cell were prepared, as previously described. ${ }^{33}$ Whole cell lysates were lysed in $1 \%$ nondietP40 lysis buffer (1\% NP-40, $150 \mathrm{mM}$ Nacl, $50 \mathrm{mM}$ Tris, pH 7.4) with freshly added protease and phosphatase inhibitors. After the lysates were incubated on ice for $30 \mathrm{~min}$, they were centrifuged $\left(12000 \times \mathrm{g}\right.$ at $\left.4{ }^{\circ} \mathrm{C}\right)$ for $5 \mathrm{~min}$ to obtain the cytosolic fraction. Protein concentrations were determined using the Bradford assay before storage at $-80^{\circ} \mathrm{C}$. Cell lysates were subjected to immunoblotting analysis using the following antibodies: anti-pAKT, anti-pGSK3 $\beta$, anti-pAMPK, anti-pJNK, antipERK, anti-pp38, anti-AKT, anti-GSK3 $\beta$, anti-AMPK, anti-JNK, anti-ERK, anti-p38, anti-Nrf-2, anti-HO-1, anti-COX-2, anti-iNOS, anti-HMGB1, anti- $\left.\right|_{\kappa} \mathrm{B} \alpha$, anti-pl $\kappa \mathrm{B} \alpha$, and anti- $\beta$-actin. The membranes were further probed with horseradish peroxidase- conjugated secondary antibodies, and detected by ECL western blot substrate. Band intensities were quantified using Image $\mathrm{J}$ gel analysis software. The fold increase in the level of protein expression was calculated by comparing it with that of normal controls. The experiments were repeated three times for each experimental condition.

Intracellular ROS measurement. To detect intracellular ROS production, RAW 264.7 cells were seeded into 12-well plates $\left(4 \times 10^{5}\right.$ cells/well) and treated with or without betulin $(9,18$ and $36 \mu \mathrm{M})$ for $1 \mathrm{~h}$, then the cells were treated with LPS $(500 \mathrm{ng} / \mathrm{ml})$ for $18 \mathrm{~h}$. Next, the cells were incubated with $50 \mu \mathrm{M}$ of DCFH-DA for $40 \mathrm{~min}$, and DCF fluorescence intensities were detected by flow cytometry.

JC-1 assay for mitochondrial membrane potential. Mitochondrial membrane potential (MMP) was measured using a mitochondrial-specific dualfluorescence probe, JC-1 (Beyotime). Briefly, RAW 264.7 cells were seeded into 12well plates $\left(4 \times 10^{5}\right.$ cells/well), treated with or without betulin $(9,18$ and $36 \mu \mathrm{M})$ for $1 \mathrm{~h}$, and then treated with LPS $(500 \mathrm{ng} / \mathrm{ml})$ for $18 \mathrm{~h}$. Next, the cells were washed with PBS and incubated with $\mathrm{JC}-1(10 \mu \mathrm{g} / \mathrm{ml})$ at $37^{\circ} \mathrm{C}$ in the dark for $30 \mathrm{~min}$. JC-1 fluorescence was quantified through flow cytometry, in which red JC-1 aggregates were gated in the FL2 channel and green JC-1 monomers in the FL1 channel.

Animal experiments. Female mice, were randomly grouped (three groups containing 10 mice) and challenged with LPS (40 mg/kg) by intraperitoneal (i.p.) injection or normal saline as a control, with two i.p. injections of Betulin (12 mg/kg), $1 \mathrm{~h}$ before and $12 \mathrm{~h}$ after the injection of LPS. Survival rates of animals were monitored every $12 \mathrm{~h}$ for 4 days.

Statistical analysis. Two-tailed unpaired Student's $t$ tests were performed to determine $P$-values. All the graphs represent the mean \pm S.E.M. of three independent experiments, and the asterisks represent $P$-values: ${ }^{*} P<0.05$, ${ }^{* \star} P<0.01$.

\section{Conflict of Interest}

The authors declare no conflicts of interest.

Acknowledgements. This work was in part supported by the National Science Foundation of China (Grant No. 81603174) and the General Financial Grant from the China Postdoctoral Science Foundation (Grant No.168847).

\section{Disclaimer}

The authors alone are responsible for the content of this manuscript.

1. Reuter S, Gupta SC, Chaturvedi MM, Aggarwal BB. Oxidative stress, inflammation, and cancer: how are they linked? Free Radic Biol Med 2010; 49: 1603-1616.

2. de Souza LF, Barreto F, da Silva EG, Andrades ME, Guimaraes EL, Behr GA et al. Regulation of LPS stimulated ROS production in peritoneal macrophages from alloxaninduced diabetic rats: involvement of high glucose and PPARgamma. Life Sci 2007; 81: 153-159.

3. Wang H, Khor TO, Saw CL, Lin W, Wu T, Huang Y et al. Role of Nrf2 in suppressing LPSinduced inflammation in mouse peritoneal macrophages by polyunsaturated fatty acids docosahexaenoic acid and eicosapentaenoic acid. Mol Pharm 2010; 7: 2185-2193.

4. de la Vega MR, Dodson M, Gross C, Manzour H, Lantz RC, Chapman E et al. Role of Nrf2 and autophagy in acute lung injury. Curr Pharmacol Rep 2016; 2: 91-101.

5. Wakabayashi N, Slocum SL, Skoko JJ, Shin S, Kensler TW. When NRF2 talks, who's listening? Antioxid Redox Signal 2010; 13: 1649-1663.

6. Kumar H, Koppula S, Kim IS, More SV, Kim BW, Choi DK. Nuclear factor erythroid 2-related factor 2 signaling in Parkinson disease: a promising multi therapeutic target against oxidative stress, neuroinflammation and cell death. CNS Neurol Disord Drug Targets 2012; 11: 1015-1029.

7. Cebula M, Schmidt EE, Arner ES. TrxR1 as a potent regulator of the Nrf2-Keap1 response system. Antioxid Redox Signal 2015; 23: 823-853.

8. Kumar H, Kim IS, More SV, Kim BW, Choi DK. Natural product-derived pharmacological modulators of Nrf2/ARE pathway for chronic diseases. Nat Prod Rep 2014; 31: 109-139.

9. Carling D, Thornton C, Woods A, Sanders MJ. AMP-activated protein kinase: new regulation, new roles? Biochem J 2012; 445: 11-27.

10. Konrad D, Rudich A, Bilan PJ, Patel N, Richardson C, Witters LA et al. Troglitazone causes acute mitochondrial membrane depolarisation and an AMPK-mediated increase in glucose phosphorylation in muscle cells. Diabetologia 2005; 48: 954-966.

11. Hayashi T, Hirshman MF, Fujii N, Habinowski SA, Witters LA, Goodyear LJ. Metabolic stress and altered glucose transport: activation of AMP-activated protein kinase as a unifying coupling mechanism. Diabetes 2000; 49: 527-531. 
12. Shirwany NA, Zou MH. AMPK: a cellular metabolic and redox sensor. A minireview. Front Biosci (Landmark Ed) 2014; 19: 447-474.

13. O'Neill LA, Hardie DG. Metabolism of inflammation limited by AMPK and pseudo-starvation. Nature 2013; 493: 346-355.

14. Levine YC, Li GK, Michel T. Agonist-modulated regulation of AMP-activated protein kinase (AMPK) in endothelial cells. Evidence for an AMPK - > Rac1 - > Akt - > endothelial nitricoxide synthase pathway. J Biol Chem 2007; 282: 20351-20364.

15. Lee J, Kim S. Upregulation of heme oxygenase-1 expression by dehydrodiconiferyl alcohol (DHCA) through the AMPK-Nrf2 dependent pathway. Toxicol Appl Pharmacol 2014; 281 87-100.

16. Zheng T, Yang X, Wu D, Xing S, Bian F, Li W et al. Salidroside ameliorates insulin resistance through activation of a mitochondria-associated AMPK/PI3K/Akt/GSK3beta pathway. $\mathrm{Br} J$ Pharmacol 2015; 172: 3284-3301.

17. Horike $\mathrm{N}$, Sakoda $\mathrm{H}$, Kushiyama $\mathrm{A}$, Ono $\mathrm{H}$, Fujishiro M, Kamata $\mathrm{H}$ et al. AMP-activated protein kinase activation increases phosphorylation of glycogen synthase kinase 3beta and thereby reduces CAMP-responsive element transcriptional activity and phosphoenolpyruvate carboxykinase C gene expression in the liver. J Biol Chem 2008; 283: 33902-33910.

18. Choi SH, Kim YW, Kim SG. AMPK-mediated GSK3beta inhibition by isoliquiritigenin contributes to protecting mitochondria against iron-catalyzed oxidative stress. Biochem Pharmacol 2010; 79: 1352-1362.

19. Mathur A, Rizvi F, Kakkar P. PHLPP2 down regulation influences nuclear Nrf2 stability via Akt-1/Gsk3beta/Fyn kinase axis in acetaminophen induced oxidative renal toxicity: Protection accorded by morin. Food Chem Toxicol 2016; 89: 19-31.

20. Xing HY, Cai YQ, Wang XF, Wang LL, Li P, Wang GY et al. The cytoprotective effect of hyperoside against oxidative stress is mediated by the Nrf2-ARE signaling pathway through GSK-3beta inactivation. PLOS ONE 2015; 10: e0145183.

21. Alakurtti S, Makela T, Koskimies S, Yli-Kauhaluoma J. Pharmacological properties of the ubiquitous natural product betulin. Eur J Pharm Sci 2006; 29: 1-13.

22. Ma C, Long H. Protective effect of betulin on cognitive decline in streptozotocin (STZ)induced diabetic rats. Neurotoxicology 2016; 57: 104-111.

23. Bai T, Yang Y, Yao YL, Sun P, Lian LH, Wu YL et al. Betulin alleviated ethanol-induced alcoholic liver injury via SIRT1/AMPK signaling pathway. Pharmacol Res 2016; 105: $1-12$.

24. Liu T, Wu L, Wang D, Wang H, Chen J, Yang $C$ et al. Role of reactive oxygen speciesmediated MAPK and NF-kappaB activation in polygonatum cyrtonema lectin-induced apoptosis and autophagy in human lung adenocarcinoma A549 cells. J Biochem 2016; 160: 315-324.
25. Roque AT, Gambeloni RZ, Felitti S, Ribeiro ML, Santos JC. Inflammation-induced oxidative stress in breast cancer patients. Med Oncol 2015; 32: 263.

26. Niture SK, Kaspar JW, Shen J, Jaiswal AK. Nrf2 signaling and cell survival. Toxicol Appl Pharmacol 2010; 244: 37-42.

27. Chunhua M, Long H, Zhu W, Liu Z, Jie R, Zhang Y et al. Betulin inhibited cigarette smokeinduced COPD in mice. Biomed Pharmacother 2017; 85: 679-686.

28. Guo MY, Li WY, Zhang Z, Qiu C, Li C, Deng G. Betulin suppresses S. aureus-induced mammary gland inflammatory injury by regulating PPAR-gamma in mice. Int Immunopharmacol 2015; 29: 824-831.

29. Zhao H, Liu Z, Liu W, Han X, Zhao M. Betulin attenuates lung and liver injuries in sepsis. Int Immunopharmacol 2016; 30: 50-56.

30. Vallabhapurapu S, Karin M. Regulation and function of NF-kappaB transcription factors in the immune system. Annu Rev Immunol 2009; 27: 693-733.

31. Guha M, Mackman N. LPS induction of gene expression in human monocytes. Cell Signal 2001; 13: 85-94.

32. de Martin R, Vanhove B, Cheng Q, Hofer E, Csizmadia V, Winkler H et al. Cytokine-inducible expression in endothelial cells of an I kappa B alpha-like gene is regulated by NF kappa B. EMBO J 1993; 12: 2773-2779.

33. Ito K, Jazrawi E, Cosio B, Barnes PJ, Adcock IM. p65-activated histone acetyltransferase activity is repressed by glucocorticoids: mifepristone fails to recruit HDAC2 to the p65-HAT complex. J Biol Chem 2001; 276: 30208-30215.

(c) Cell Death and Disease is an open-access journal published by Nature Publishing Group. This work is licensed under a Creative Commons Attribution 4.0 International License. The images or other third party material in this article are included in the article's Creative Commons license, unless indicated otherwise in the credit line; if the material is not included under the Creative Commons license, users will need to obtain permission from the license holder to reproduce the material. To view a copy of this license, visit http://creativecommons.org/licenses/by/4.0/

(C) The Author(s) 2017 